\title{
Pruritus in autoimmune connective tissue diseases
}

\author{
Hee Joo Kim \\ Department of Dermatology, Gachon Gil Medical Center, Gachon University College of Medicine, Incheon, South Korea \\ Correspondence to: Hee Joo Kim, MD, PhD. 21, Namdongdae-ro 774beon-gil, Namdong-gu, Incheon, 21565, South Korea. Email: hjbp0610@gachon.ac.kr.
}

\begin{abstract}
Pruritus is one of the most common and bothersome symptoms of skin disorders, and its clinical characteristics and related pathomechanisms have been well described in certain dermatologic conditions, such as atopic dermatitis and urticaria. Although pruritus is believed to be as common in cutaneous autoimmune connective tissue diseases (ACTDs) as in other inflammatory skin disorders, its true characteristics have not been elucidated either qualitatively or quantitatively. Pruritus is present in ACTDs with various prevalence rates, characteristics, and mechanisms depending on the disease types. Pruritus most frequently and severely affects the patients with dermatomyositis, in which itch is strongly correlated with disease activity and severity, thus increased itch could also indicate a disease flare. Patients with other ACTDs, including lupus erythematosus (LE), Sjögren syndrome (SS), morphea, and systemic sclerosis (SSc), also suffer from their fair share of pruritus. Unfortunately, the currently available treatments for ACTDs seem to have only limited and unsatisfactory effects to control pruritus. The extensive impact of pruritus on the patients' quality of life (QOL) and functioning warrants more targeted and individualized approaches against pruritus in ACTDs. This review will address the prevalence, suggested pathogenesis based on currently available evidences, and potential treatment options of pruritus in various ACTDs of the skin.
\end{abstract}

Keywords: Autoimmune connective tissue disease (ACTD); itch; pathogenesis; pruritus

Submitted Jun 23, 2020. Accepted for publication Dec 31, 2020.

doi: 10.21037/atm-20-4894

View this article at: http://dx.doi.org/10.21037/atm-20-4894

Pruritus, an unpleasant sensation leading to a scratch response, is one of the most crucial components of nociception (1). It is considered the most common symptom associated with skin disorders, reported in more than half of the patients (2). The clinical characteristics and related pathomechanisms of pruritus have been extensively explored and well elucidated in certain dermatologic conditions, such as atopic dermatitis and urticaria, whereas the true impact of itch in other diseases such as psoriasis has only been recently investigated (3). Autoimmune connective tissue diseases (ACTDs) represent various immune dysregulationrelated multisystemic disorders with heterogeneous but overlapping clinical manifestations, and frequently involve the skin. Cutaneous ACTDs, including lupus erythematosus (LE), dermatomyositis, Sjögren syndrome (SS), morphea, and systemic sclerosis (SSc), are no exceptions to having pruritus as one of the prominent symptoms. Among the reported patients with ACTDs, regardless of the diagnosis, $57 \%$ complained of pruritus during the initial visit (4). Skin symptoms significantly affect the patients' quality of life (QOL), and pruritus substantially contribute to the impact (5). However, pruritus has not been well described either qualitatively or quantitatively in ACTDs, and remains a nettlesome puzzle to both patients and physicians. This review will address the prevalence, currently suggested pathogenesis, and potential treatment of itch in various ACTDs of the skin.

\section{Dermatomyositis}

Dermatomyositis is by far the most conspicuously pruritus-affected ACTD, even more than other common inflammatory skin disorders such as atopic dermatitis or psoriasis, and other ACTDs such as LE (6-10). The prevalence of itch in dermatomyositis is usually reported to be higher than $50 \%$, varying depending on the study population $(11,12)$. In a study cohort of 191 patients, more than $90 \%$ of the patients had pruritus and $50.8 \%$ 
had moderate-to-severe itch, and the itch severity was correlated with increased cutaneous severity independent of muscle involvement (13). Pruritus in dermatomyositis was reported to be persistent from the time of diagnosis, even when muscle involvement was under control (4). Pruritus is commonly located on the arms and trunk, face, and scalp, consistent with the typical location of skin involvement in dermatomyositis. Pruritus on the scalp is more persistent and recurrent, possibly owing to greater exposure to the sun and difficulties in topical application of medication. Pruritus was reported to be less frequent in juvenile dermatomyositis than in the adult population (38\%) (14). In dermatomyositis, QOL impairment is greater than in other skin conditions including psoriasis and atopic dermatitis, and better QOL is associated with improved itch and skin symptoms $(5,15)$. In a study using a $100-\mathrm{mm}$ visual analog scale (VAS) questionnaire, most patients had noticeable pruritus with a mean score of $>50$ on the VAS, and a mean of 44.6 was found for the effect on daily life (16). Considering its high prevalence and severity in dermatomyositis, several classification criteria for amyopathic dermatomyositis include pruritus as a component $(7,8)$. In the absence of typical muscle symptoms, intense pruritus can cause the misdiagnosis of amyopathic dermatomyositis as other inflammatory or allergic diseases; thus, clinicians need to be aware of the importance of pruritus in dermatomyositis (17).

Little is known about the pathogenesis of itch in dermatomyositis. One potential mediator is interleukin (IL)-31, which has been implicated in pruritus associated with various pruritic skin diseases, such as atopic dermatitis, prurigo nodularis, and cutaneous T-cell lymphoma $(18,19)$. Skin IL-31 and its receptor, IL-31 receptor alpha, were significantly upregulated in dermatomyositis, and IL-31 mRNA expression was positively correlated with the VAS itch score $(\mathrm{r}=0.67)$, indicating that IL-31 may play a role in the pathogenesis of dermatomyositis-related pruritus. CD4+ $\mathrm{T}$ cells were the most abundant IL-31-producing cell type, whereas other cell types expressing CD8, CD68, CD11b, or CD11c were also found to secrete IL-31 in dermatomyositis (13).

Small fiber neuropathies (SFNs) are another suggested mechanism for the itch in dermatomyositis. SFNs are a recently described disease set of small nerve fibers and a major cause of neuropathic itch (20). As small nerve fibers are abundant in the skin and mucosa, clinical symptoms of SFNs, including autonomic symptoms such as red or white skin discoloration and sensory symptoms such as pruritus, burning, or tingling, are frequently found in the skin. With reduced intraepidermal nerve fiber density being a major diagnostic criterion of SFNs, these diseases are implicated in various skin disorders that feature intractable pruritus, including prurigo nodularis and nummular eczema (21-24). Interestingly, significantly reduced epidermal nerve fiber density with abnormal morphology and complex cluster has been found in lesional biopsy of the treatment-refractory itchy scalp of a patient with dermatomyositis (25). Although this finding has not been re-confirmed in a larger number of patients, further mechanistic studies would be necessary to find the true meaning of SFNs in dermatomyositis.

Comorbidities of dermatomyositis can also contribute to pruritus in dermatomyositis. Adult dermatomyositis frequently accompanies malignancy, and chronic pruritus could be an initial sign of malignancy. Thus, paraneoplastic itch should be considered in adult patients with dermatomyositis, particularly in those with clinical symptoms suggestive of malignancy $(11,26)$.

Currently, there is no gold standard for managing the incessant pruritus in dermatomyositis. The co-localization of itch and skin inflammatory lesions suggests an inflammatory cause of pruritus, thus warranting immunosuppressives as pruritus-targeting therapies. However, conventional antiinflammatory regimens for dermatomyositis occasionally show unsatisfactory results, particularly for pruritus on the scalp. The treatments with reported anecdotal successes include apremilast for severe scalp pruritus (27), dapsone for itchy poikilodermatous eruption in dermatomyositis (28), high-dose intravenous immunoglobulin for pruritic skin lesion of malignancy-associated dermatomyositis (29), and low-dose naltrexone for pruritus and burning of the scalp (30).

Recently, lenabasum, a synthetic, nonimmunosuppressive, non-psychoactive selective cannabinoid receptor type 2 agonist, has been shown to have acceptable safety and tolerability and improved efficacy in a phase 2 trial (NCT02466243) and open-label extension study in patients with dermatomyositis with refractory, skin-predominant involvement (31). Lenabasum significantly downregulated IL-31 from CpG-stimulated peripheral blood mononuclear cells, implying its potential role in controlling pruritus in dermatomyositis (13). This cannabinoid has also been shown to reduce other inflammatory cytokines, such as tumor necrosis factor- $\alpha$, type I interferons, and IL-4, in dermatomyositis in vitro $(13,32)$. A phase 3 multicenter clinical trial evaluating the efficacy and safety of lenabasum in dermatomyositis is ongoing (33). 


\section{Cutaneous lupus erythematosus (CLE)}

In contrast to dermatomyositis, pruritus in CLE has limited and controversial data. Pruritus is usually considered less frequent and less severe in CLE than in dermatomyositis $(4,9)$. The median pruritus VAS score was reported to be appreciably higher in dermatomyositis than in CLE (3.8 vs. 2.0) $(\mathrm{P}<0.0001)$ (9). In an observational study analyzing the correlation of change in the CLE Disease Area and Severity Index (CLASI) score with the change in patients' assessments of pain and pruritus after therapy, the correlation of CLASI score with the patients' assessment of itch was only moderate (34). Itch was not present in every patient, and some patients developed pruritus regardless of the skin condition and treatment. However, when pruritus was evaluated in systemic LE with skin symptoms, the overall median VAS score for pruritus was 6 (range, 0-9) (35). The pruritus score did not differ depending on the CLE lesion types, which were classified into specific and nonspecific according to the presence of LE-specific histologic features. In the group with specific lesions, pruritus and the CLASI activity score were correlated $(\tau=0.57, \mathrm{P}=0.01$ ), although the overall pruritus score did not correlate with the QOL evaluated using the Dermatology Life Quality Index (DLQI) or the CLASI activity score.

However, patients with CLE also suffer from pruritus. In a retrospective study, pruritus in lupus was closely related to skin disease activity, and pruritus was associated with disease flare and sun exposure in $45 \%$ (4). The severity of itch was mainly mild (45\%) to moderate (54\%). In a recent multinational multicenter cross-sectional study, pruritus was present in $75 \%$ of patients with CLE, and was most frequent in acute CLE (82.1\%) followed by chronic CLE (78.8\%) (36). On the basis of the numerical rating scale, the severity of itch was mostly in the mild category (62.1\%).

Studies evaluating the therapeutic efficacy against pruritus in CLE are surprisingly scarce. As immunosuppression is the mainstay treatment for CLE to reduce inflammation, it can also aid in decreasing pruritus (37). Topical agents, including corticosteroids and calcineurin inhibitors, and antimalarials, are the most commonly used treatments, although some patients with more severe disease need systemic treatment with oral corticosteroids or steroid-sparing agents, such as methotrexate, mycopheolate mofetil, azathioprine, and thalidomide. However, more studies are required to measure the actual effect of the treatments for controlling pruritus in CLE.

\section{SSc}

Abnormal fibrosis of the skin and internal organs with vasculopathy characterizes SSc. The Canadian Scleroderma Research Group reported that more than $40 \%$ of patients with SSc complained of pruritus, and patients with pruritus had more severe skin disease, worse respiratory symptoms, and greater gastrointestinal tract involvements $(38,39)$. A questionnaire-based study from France reported a higher prevalence of pruritus (62\%) in patients with SSc (40). Pruritus was worsened by fatigue, stress, xerosis, and specific clothing materials, whereas rest, sleep, hot water, and hot ambient temperature were slightly helpful in alleviating pruritus. About $40 \%$ of patients with pruritus had pruritus on the skin affected by SSc, most commonly on the head, followed by the back and the upper and lower extremities. The urge to scratch, pruritus-induced burning, or stinging were the pruritus-associated symptoms with the greatest impact on the QOL. In another study on chronic pruritus in SSc, chronic itch was present in $56.7 \%$ of the patients with SSc (41). Chronic itch was considerably associated with skin dryness, and the back was the most commonly affected location, followed by the arms and scalp. The patients described their pruritus as prickling, painful, burning, and stinging. Itch was not significantly associated with the presence of autoantibodies, such as anti-nuclear antibodies, anti-Scl-70 antibodies, and anti-centromere antibodies (ACAs). It was also not related to the involvement of internal organs, including the lung, kidney, gastrointestinal system, or joints. Pruritus also compromises the functioning of patients with SSc, both mentally and physically (42-44). Those with more severe pain and itch are more likely to experience greater depressive symptoms, sleep problems and fatigue, and overall disability problems (44). The severity of pruritus has also been reported to be independently associated with greater sleep disturbance, along with the number of gastrointestinal symptoms and the pain severity (45).

The pathogenesis of pruritus in SSc is still not clearly identified. Pruritus is not specifically associated with a particular immunological profile, whereas the autoimmune status has been associated with the characteristics of pruritus in SSc. The presence of ACA has been reported to be associated with the duration, location, and appearance order of pruritus against skin symptoms (46). In that study, $\mathrm{ACA}+$ patients had a longer period of pruritus than the ACA- subgroup, and pruritus predominantly occurred in nonsclerotic areas (82.4\%). Conversely, sclerotic areas 
(63.2\%) were more greatly affected by pruritus in ACApatients. In the ACA+ subgroup, pruritus occurred before SSc in $17.6 \%$, pruritus and skin sclerosis concomitantly appeared in $47.1 \%$, and pruritus and skin sclerosis appeared in subsequent order in $35.3 \%$ of the cases. Conversely, in the ACA- subgroup, both events were predominantly concomitant in $84.2 \%$ of the cases.

The endocannabinoid system is another possible intervening system for pruritus in SSc. Endocannabinoids originally drew attention in SSc for their potential antifibrotic effects. The cannabinoid receptor CB2 has been demonstrated to exert anti-fibrotic effects in experimental dermal fibrosis (47). In that study, CB2 knockout mice or wild-type mice treated with a CB2 antagonist were more susceptible to bleomycin-induced dermal fibrosis than controls, and their dermal thickness and leukocyte counts in lesional skin were significantly increased, which were decreased by treatment with a CB2 agonist. Interestingly, the endocannabinoid system is important in central and peripheral processing of skin-originated pain and itch. Synthetic cannabinoid receptor agonists and/ or endocannabinoids exhibit potent analgesic effects by activating not only cannabinoid receptors but also possibly other receptors, such as TRPV1, in sensory neurons and inflammatory cells (48-50). They are believed to suppress the release of pruritogenic substances and to inhibit the transmission of signals in the nervous system.

Various pruritogens, including lysophosphatidic acid (LPA), have been vaguely linked to pruritus in SSc. Increased LPA near unmyelinated nerve endings has been suggested to cause itch, and LPA has been reported to be elevated in SSc (51-53). As patients often experience pruritus associated with neuropathic symptoms, nerve fiber damage may also contribute to the itch in SSc (40).

Considering that SSc features irreversible damage to the normal adnexal structures as well as the underlying inflammation, immunosuppressive and anti-inflammatory agents may not be sufficient to relieve pruritus in SSc (41). As expected, it is also refractory to anti-histamines, the most commonly used medication for controlling pruritus (51). In a double-blind, randomized controlled trial of ketotifen in early diffuse SSc based on the potential role of mast cells in fibrosis of SSc, pruritus (evaluated using a four-point scale, $0-3)$ tended to improve more in patients who took ketotifen $(2.8 \pm 1.1$ at baseline to $1.5 \pm 1.5$ at week 24$)$ than in those who took placebo $(1.9 \pm 1.4$ to $1.3 \pm 1.8)$, although statistical significance was not reported (54). Only one case series has evaluated the therapeutic efficacy for pruritus in SSc, and low-dose naltrexone was suggested as a tolerable option (51). Among three patients with itchy SSc, two patients reported complete resolution of pruritus after low-dose naltrexone and the third patient also reported improvement of pruritus according to a 10-point faces scale. Given the associated neuropathic symptoms, exploring the therapeutic potential of neuroactive medications for controlling neuropathic itch, such as gabapentin or pregabalin, is warranted.

\section{Morphea}

Morphea is clinically distinct from SSc, manifesting as localized plaques limited to the skin without systemic involvement; however, it is histopathologically indistinguishable from SSc featuring sclerotic dermis. However, the itch in morphea is much less vigorously studied. In a morphea cohort, $78.3 \%$ of the patients reported at least one symptom, most commonly itch followed by pain (55). In another cross-sectional study, $46 \%$ of the subjects reported itchy or painful skin (56).

Pruritus is associated with impairment in skin-specific and global health-related QOL (HRQOL), and negatively affects QOL both in adults and children $(55,57)$. Itchy or painful skin $(46 \%)$ and clothing issues (43\%) were the most commonly reported problems in a study using the DLQI (56). Pain and itch were found to be even stronger correlates of HRQOL than the location of lesions in cosmetically or functionally sensitive sites (57). Itch was also significantly correlated with lesion activity, suggesting that pruritus may be a marker of active disease $(55,57)$.

As the pathogenesis of pruritus in morphea is still elusive, efforts to control morphea-specific pruritus are also sparse. In a previous study, phototherapy significantly decreased the VAS itch score with medium-dose ultraviolet (UV)-A1, but not with low-dose UVA1 and narrow-band UVB (58). In another study, although the effect on pruritus was not specifically measured, medium- to high-dose UVA1 phototherapy was effective in reducing the clinical score of morphea, based on body surface area, erythema, induration, sclerosis, pigmentation, and symptoms of pain or pruritus (59).

\section{SS}

The major skin symptoms of SS include dry skin, eyelid dermatitis, angular cheilitis, annular erythema, and vasculitis (60). The absence of an associated disease defines primary SS, whereas its presence indicates secondary SS. 
An Italian group reported that pruritus was common in both primary SS (41.9\%) and secondary SS (38.3\%) (60). In another cohort of primary SS, chronic pruritus with a mean duration of 74.4 months was present in $53 \%$ of the subjects (61). The mean VAS itch scale was 7.7, and xerosis was more common in itchy patients (90\%) than in non-itchy patients (44\%). Itch was most common on the shin $(90 \%)$, followed by the back (80\%) and forearm (70\%). The most common aggravating factors were xerosis (70\%) and hot water $(50 \%)$. The patients reported that the itch was more severe in the evening and during the summer. In that study, QOL was significantly affected by pruritus, proportionate to the severity of itch. The impact was profound in the emotional domain of the ItchyQoL, in which the effect of itch on the QOL is analyzed in the three domains of symptoms, functional limitations, and emotions related to itch (62). The patients reported that the itch was worsened by temperature or seasonal changes, resulting in the need to scratch (61). However, their pruritus was independent of liver dysfunction or cholestasis, a common systemic cause of pruritus (63).

The mechanism of itch in SS is not well understood; however, dry skin, which is a common cause of chronic itch in other dermatologic conditions such as atopic dermatitis or elderly skin, is also a main characteristic of SS $(60,64)$. Patients' self-reports of dryness-induced worsening of pruritus also support the role of xerosis in SS-associated pruritus. Meanwhile, the reason why itch intensifies during the summer, when dry skin usually improves, has been suggested to be associated with sweat gland destruction found in SS, because anhidrotic patients tend to experience pruritus along with heat (61).

Another noteworthy link is concurrent primary biliary cirrhosis (PBC) $(65,66)$. Dry eyes and dry mouth, which are the most common symptoms of SS, are similarly common in patients with PBC (67). Several studies have shown an association between $\mathrm{PBC}$ and $\mathrm{SS}$, with reported SS prevalence of about $34 \%$ in PBC (65). Pruritus and fatigue are the most common symptoms of PBC, both independent of disease severity (68). Although ursodeoxycholic acid is the essential treatment for all patients with PBC, this treatment does not affect the symptoms of fatigue and pruritus.

Unfortunately, there has been no specific therapeutic approach to improve HRQOL in an adequately powered double-blind, placebo-controlled randomized controlled trial (69). The lack of response of pruritus in SS to immunosuppressives suggests that pruritus may be more related to disease-related damage than to inflammation; thus, alternative therapeutics, such as neuromodulators, may be necessary (4). SS has been implicated as a rare cause of brachial and lumbosacral plexopathies, as well as ganglionopathy, implying that itch in SS may have a neuropathic etiology $(70,71)$.

\section{Antimalarial medication-induced pruritus}

Another factor that should not be overlooked is drugrelated pruritus, particularly when the underlying disease is well controlled but patients still complain of refractory pruritus. Antimalarials remain a mainstay treatment for ACTDs, particularly LE, thanks to their anti-inflammatory effects and low adverse effect profile (72). However, in a review of 209 patients receiving antimalarial treatment, xerosis followed by hyperpigmentation and pruritus were frequently observed, although the prevalence of pruritus was not statistically significant $(\mathrm{P}<0.39)$ compared with the control group (73). In a case report, pruritus had a strong temporal association with the hydroxychloroquine challenge (74). In addition to non-triggered itch, aquagenic itch has been reported in association with antimalarials (75). Interestingly, antimalarial-associated pruritus seems to vary depending on the specific drug types. In some cases, switching from one antimalarial to another can reduce or eliminate the adverse effects. Adverse cutaneous reactions to hydroxychloroquine are more common in patients with dermatomyositis than in those with cutaneous lupus (76). Pruritus is a major adverse effect of chloroquine use and is an important cause of noncompliance, especially in Africans compared with Caucasians (77). It has been shown that chloroquine stimulates the itch nerves by activating the $G$ protein-coupled receptor Mrgpr, expressed exclusively in peripheral sensory neurons (78). Mrgpr genes are highly polymorphic, which underlies the ethnic variation in chloroquine-induced itch.

\section{Conclusions}

Pruritus is common in ACTDs with various prevalence rates, characteristics, and mechanisms depending on the disease types. For some diseases in which itch is strongly correlated with disease activity and severity, including dermatomyositis, increased itch could also indicate a disease flare. The extensive impact of pruritus on the patients' QOL and functioning warrants more targeted and individualized approaches against pruritus in ACTDs. 


\section{Acknowledgments}

Funding: None.

\section{Footnote}

Provenance and Peer Review: This article was commissioned by the Guest Editors (Drs. Richard D. Sontheimer, M. Kari Connolly, David F. Fiorentino, and Victoria P. Werth) for the series "Rheumatologic Skin Disease" published in Annals of Translational Medicine. The article has undergone external peer review.

Conflicts of Interest: The author has completed the ICMJE uniform disclosure form (available at http://dx.doi. org/10.21037/atm-20-4894). The series "Rheumatologic Skin Disease" was commissioned by the editorial office without any funding or sponsorship. The author has no other conflicts of interest to declare.

Ethical Statement: The author is accountable for all aspects of the work in ensuring that questions related to the accuracy or integrity of any part of the work are appropriately investigated and resolved.

Open Access Statement: This is an Open Access article distributed in accordance with the Creative Commons Attribution-NonCommercial-NoDerivs 4.0 International License (CC BY-NC-ND 4.0), which permits the noncommercial replication and distribution of the article with the strict proviso that no changes or edits are made and the original work is properly cited (including links to both the formal publication through the relevant DOI and the license). See: https://creativecommons.org/licenses/by-nc-nd/4.0/.

\section{References}

1. Yosipovitch G, Greaves MW, Schmelz M. Itch. Lancet 2003;361:690-4.

2. Schut C, Dalgard FJ, Halvorsen JA, et al. Occurrence, Chronicity and Intensity of Itch in a Clinical Consecutive Sample of Patients with Skin Diseases: A Multi-centre Study in 13 European Countries. Acta Derm Venereol 2019;99:146-51.

3. Szepietowski JC, Reich A, Wisnicka B. Itching in patients suffering from psoriasis. Acta Dermatovenerol Croat 2002;10:221-6.

4. Yahya A, Gideon PS. Characterizing Pruritus in
Autoimmune Connective Tissue Diseases. J Drugs Dermatol 2019;18:995-8.

5. Hundley JL, Carroll CL, Lang W, et al. Cutaneous symptoms of dermatomyositis significantly impact patients' quality of life. J Am Acad Dermatol 2006;54:217-20.

6. Concha JSS, Tarazi M, Kushner CJ, et al. The diagnosis and classification of amyopathic dermatomyositis: a historical review and assessment of existing criteria. $\mathrm{Br} \mathrm{J}$ Dermatol 2019;180:1001-8.

7. Dalakas MC, Hohlfeld R. Polymyositis and dermatomyositis. Lancet 2003;362:971-82.

8. Sontheimer RD. Dermatomyositis: an overview of recent progress with emphasis on dermatologic aspects. Dermatol Clin 2002;20:387-408.

9. Goreshi R, Chock M, Foering K, et al. Quality of life in dermatomyositis. J Am Acad Dermatol 2011;65:1107-16.

10. Sontheimer RD. Cutaneous features of classic dermatomyositis and amyopathic dermatomyositis. Curr Opin Rheumatol 1999;11:475-82.

11. Yosipovitch G, Tan A, LoSicco K, et al. A comparative study of clinical characteristics, work-up, treatment, and association to malignancy in dermatomyositis between two tertiary skin centers in the USA and Singapore. Int J Dermatol 2013;52:813-9.

12. Galimberti F, Li Y, Fernandez AP. Clinically amyopathic dermatomyositis: clinical features, response to medications and malignancy-associated risk factors in a specific tertiarycare-centre cohort. Br J Dermatol 2016;174:158-64.

13. Kim HJ, Zeidi M, Bonciani D, et al. Itch in dermatomyositis: the role of increased skin interleukin-31. Br J Dermatol 2018;179:669-78.

14. Peloro TM, Miller OF 3rd, Hahn TF, et al. Juvenile dermatomyositis: a retrospective review of a 30-year experience. J Am Acad Dermatol 2001;45:28-34.

15. Robinson ES, Feng R, Okawa J, et al. Improvement in the cutaneous disease activity of patients with dermatomyositis is associated with a better quality of life. Br J Dermatol 2015;172:169-74.

16. Shirani Z, Kucenic MJ, Carroll CL, et al. Pruritus in adult dermatomyositis. Clin Exp Dermatol 2004;29:273-6.

17. Poddighe D, Mukusheva Z, Assylbekova M. Pruritus in patients with amyopathic dermatomyositis. Allergy Asthma Clin Immunol 2019;15:18.

18. Nattkemper LA, Martinez-Escala ME, Gelman AB, et al. Cutaneous T-cell Lymphoma and Pruritus: The Expression of IL-31 and its Receptors in the Skin. Acta Derm Venereol 2016;96:894-8.

19. Sonkoly E, Muller A, Lauerma AI, et al. IL-31: a new link 
between $\mathrm{T}$ cells and pruritus in atopic skin inflammation. $\mathrm{J}$ Allergy Clin Immunol 2006;117:411-7.

20. Oaklander AL. Neuropathic itch. Semin Cutan Med Surg 2011;30:87-92.

21. Devigili G, Tugnoli V, Penza P, et al. The diagnostic criteria for small fibre neuropathy: from symptoms to neuropathology. Brain 2008;131:1912-25.

22. Maddison B, Parsons A, Sangueza O, et al. Retrospective study of intraepidermal nerve fiber distribution in biopsies of patients with nummular eczema. Am J Dermatopathol 2011;33:621-3.

23. Schuhknecht B, Marziniak M, Wissel A, et al. Reduced intraepidermal nerve fibre density in lesional and nonlesional prurigo nodularis skin as a potential sign of subclinical cutaneous neuropathy. Br J Dermatol 2011;165:85-91.

24. Tavee J, Zhou L. Small fiber neuropathy: A burning problem. Cleve Clin J Med 2009;76:297-305.

25. Hurliman E, Groth D, Wendelschafer-Crabb G, et al. Small-fibre neuropathy in a patient with dermatomyositis and severe scalp pruritus. Br J Dermatol 2017;176:209-11.

26. Yosipovitch G. Chronic pruritus: a paraneoplastic sign. Dermatol Ther 2010;23:590-6.

27. Charlton D, Moghadam-Kia S, Smith K, et al. Refractory Cutaneous Dermatomyositis With Severe Scalp Pruritus Responsive to Apremilast. J Clin Rheumatol 2019.

28. Kawachi Y, Fujisawa Y, Furuta J, et al. Pruritic poikilodermatous eruption associated with dermatomyositis: successful treatment with dapsone. Eur J Dermatol 2012;22:289-90.

29. Kikuchi-Numagami K, Sato M, Tagami H. Successful treatment of a therapy-resistant severely pruritic skin eruption of malignancy-associated dermatomyositis with high-dose intravenous immunoglobulin. J Dermatol 1996;23:340-3.

30. Manudhane AP, Schrom KP, Ezaldein HH, et al. Lowdose naltrexone: a unique treatment for amyopathic dermatomyositis. Dermatol Online J 2019;25.

31. Werth VP, Pearson DR, Okawa J, et al. Safety and Efficacy of Lenabasum in Refractory Skin-Predominant Dermatomyositis Subjects Treated on an Open-Label Extension of Trial JBT101-DM-001 [abstract]. Arthritis Rheumatol 2018;70.

32. Robinson ES, Alves P, Bashir MM, et al. Cannabinoid Reduces Inflammatory Cytokines, Tumor Necrosis Factoralpha, and Type I Interferons in Dermatomyositis In Vitro. J Invest Dermatol 2017;137:2445-7.

33. ClinicalTrials.gov. Available online: https://clinicaltrials.
gov/ct2/show/NCT03813160. Accessed 2020-01-07.

34. Bonilla-Martinez ZL, Albrecht J, Troxel AB, et al. The cutaneous lupus erythematosus disease area and severity index: a responsive instrument to measure activity and damage in patients with cutaneous lupus erythematosus. Arch Dermatol 2008;144:173-80.

35. Méndez-Flores S, Orozco-Topete R, Bermudez-Bermejo $\mathrm{P}$, et al. Pain and pruritus in cutaneous lupus: their association with dermatologic quality of life and disease activity. Clin Exp Rheumatol 2013;31:940-2.

36. Samotij D, Szczech J, Kushner CJ, et al. Prevalence of Pruritus in Cutaneous Lupus Erythematosus: Brief Report of a Multicenter, Multinational Cross-Sectional Study. Biomed Res Int 2018;2018:3491798.

37. Smith GP, Argobi Y. Pruritus in Autoimmune Connective Tissue Diseases. Dermatol Clin 2018;36:267-75.

38. Razykov I, Levis B, Hudson M, et al. Prevalence and clinical correlates of pruritus in patients with systemic sclerosis: an updated analysis of 959 patients. Rheumatology (Oxford) 2013;52:2056-61.

39. Razykov I, Thombs BD, Hudson M, et al. Prevalence and clinical correlates of pruritus in patients with systemic sclerosis. Arthritis Rheum 2009;61:1765-70.

40. Théréné C, Brenaut E, Sonbol H, et al. Itch and systemic sclerosis: frequency, clinical characteristics and consequences. Br J Dermatol 2017;176:1392-3.

41. Stull CM, Weaver LA, Valdes-Rodriguez R, et al. Characteristics of Chronic Itch in Systemic Sclerosis: A Cross-sectional Survey. Acta Derm Venereol 2018;98:793-4.

42. Chularojanamontri L, Sethabutra P, Kulthanan K, et al. Dermatology life quality index in Thai patients with systemic sclerosis: a cross-sectional study. Indian J Dermatol Venereol Leprol 2011;77:683-7.

43. El-Baalbaki G, Razykov I, Hudson M, et al. Association of pruritus with quality of life and disability in systemic sclerosis. Arthritis Care Res (Hoboken) 2010;62:1489-95.

44. Racine $M$, Hudson M, Baron M, et al. The Impact of Pain and Itch on Functioning and Health-Related Quality of Life in Systemic Sclerosis: An Exploratory Study. J Pain Symptom Manage 2016;52:43-53.

45. Milette K, Hudson M, Korner A, et al. Sleep disturbances in systemic sclerosis: evidence for the role of gastrointestinal symptoms, pain and pruritus. Rheumatology (Oxford) 2013;52:1715-20.

46. Gourier G, Therene C, Mazeas M, et al. Clinical Characteristics of Pruritus in Systemic Sclerosis Vary According to the Autoimmune Subtype. Acta Derm 
Venereol 2018;98:735-41.

47. Akhmetshina A, Dees C, Busch N, et al. The cannabinoid receptor $\mathrm{CB} 2$ exerts antifibrotic effects in experimental dermal fibrosis. Arthritis Rheum 2009;60:1129-36.

48. Bíró T, Toth BI, Hasko G, et al. The endocannabinoid system of the skin in health and disease: novel perspectives and therapeutic opportunities. Trends Pharmacol Sci 2009;30:411-20.

49. Walker JM, Hohmann AG. Cannabinoid mechanisms of pain suppression. Handb Exp Pharmacol 2005:509-54.

50. Ashton JC, Milligan ED. Cannabinoids for the treatment of neuropathic pain: clinical evidence. Curr Opin Investig Drugs 2008;9:65-75.

51. Frech T, Novak K, Revelo MP, et al. Low-dose naltrexone for pruritus in systemic sclerosis. Int J Rheumatol 2011;2011:804296.

52. Oude Elferink RP, Kremer AE, Beuers U. Mediators of pruritus during cholestasis. Curr Opin Gastroenterol 2011;27:289-93.

53. Tokumura A, Carbone LD, Yoshioka Y, et al. Elevated serum levels of arachidonoyl-lysophosphatidic acid and sphingosine 1-phosphate in systemic sclerosis. Int J Med Sci 2009;6:168-76.

54. Gruber BL, Kaufman LD. A double-blind randomized controlled trial of ketotifen versus placebo in early diffuse scleroderma. Arthritis Rheum 1991;34:362-6.

55. Das S, Bernstein I, Jacobe H. Correlates of self-reported quality of life in adults and children with morphea. J Am Acad Dermatol 2014;70:904-10.

56. Bali G, Karpati S, Sardy M, et al. Association between quality of life and clinical characteristics in patients with morphea. Qual Life Res 2018;27:2525-32.

57. Klimas NK, Shedd AD, Bernstein IH, et al. Healthrelated quality of life in morphoea. Br J Dermatol 2015;172:1329-37.

58. Kreuter A, Hyun J, Stucker M, et al. A randomized controlled study of low-dose UVA1, medium-dose UVA1, and narrowband UVB phototherapy in the treatment of localized scleroderma. J Am Acad Dermatol 2006;54:440-7.

59. Jacobe HT, Cayce R, Nguyen J. UVA1 phototherapy is effective in darker skin: a review of 101 patients of Fitzpatrick skin types I-V. Br J Dermatol 2008;159:691-6.

60. Bernacchi E, Amato L, Parodi A, et al. Sjogren's syndrome: a retrospective review of the cutaneous features of 93 patients by the Italian Group of Immunodermatology. Clin Exp Rheumatol 2004;22:55-62.
61. Valdes-Rodriguez R, Rowe B, Lee HG, et al. Chronic Pruritus in Primary Sjogren's Syndrome: Characteristics and Effect on Quality of Life. Acta Derm Venereol 2017;97:385-6.

62. Desai NS, Poindexter GB, Monthrope YM, et al. A pilot quality-of-life instrument for pruritus. J Am Acad Dermatol 2008;59:234-44.

63. Düll MM, Kremer AE. Treatment of Pruritus Secondary to Liver Disease. Curr Gastroenterol Rep 2019;21:48.

64. Engelke M, Jensen JM, Ekanayake-Mudiyanselage S, et al. Effects of xerosis and ageing on epidermal proliferation and differentiation. Br J Dermatol 1997;137:219-25.

65. Floreani A, Franceschet I, Cazzagon N, et al. Extrahepatic autoimmune conditions associated with primary biliary cirrhosis. Clin Rev Allergy Immunol 2015;48:192-7.

66. Corpechot C, Chretien Y, Chazouilleres O, et al. Demographic, lifestyle, medical and familial factors associated with primary biliary cirrhosis. J Hepatol 2010;53:162-9.

67. Lindor KD, Gershwin ME, Poupon R, et al. Primary biliary cirrhosis. Hepatology 2009;50:291-308.

68. Carey EJ, Ali AH, Lindor KD. Primary biliary cirrhosis. Lancet 2015;386:1565-75.

69. Miyamoto ST, Valim V, Fisher BA. Health-related quality of life and costs in Sjogren's syndrome. Rheumatology (Oxford) 2019;key370.

70. Griffin JW, Cornblath DR, Alexander E, et al. Ataxic sensory neuropathy and dorsal root ganglionitis associated with Sjogren's syndrome. Ann Neurol 1990;27:304-15.

71. Misery L, Brenaut E, Le Garrec R, et al. Neuropathic pruritus. Nat Rev Neurol 2014;10:408-16.

72. Mittal L, Zhang L, Feng R, et al. Antimalarial drug toxicities in patients with cutaneous lupus and dermatomyositis: A retrospective cohort study. J Am Acad Dermatol 2018;78:100-6.e1.

73. Skare T, Ribeiro CF, Souza FH, et al. Antimalarial cutaneous side effects: a study in 209 users. Cutan Ocul Toxicol 2011;30:45-9.

74. Gül U, Cakmak SK, Kilic A, et al. A case of hydroxychloroquine induced pruritus. Eur J Dermatol 2006;16:586-7.

75. Jiménez-Alonso J, Tercedor J, Jaimez L, et al. Antimalarial drug-induced aquagenic-type pruritus in patients with lupus. Arthritis Rheum 1998;41:744-5.

76. Pelle MT, Callen JP. Adverse cutaneous reactions to hydroxychloroquine are more common in patients with dermatomyositis than in patients with cutaneous 
lupus erythematosus. Arch Dermatol 2002;138:1231-3; discussion 1233.

77. Tey HL, Yosipovitch G. Itch in ethnic populations. Acta Derm Venereol 2010;90:227-34.

Cite this article as: Kim HJ. Pruritus in autoimmune connective tissue diseases. Ann Transl Med 2021;9(5):441. doi: 10.21037/atm-20-4894
78. Liu Q, Tang Z, Surdenikova L, et al. Sensory neuronspecific GPCR Mrgprs are itch receptors mediating chloroquine-induced pruritus. Cell 2009;139:1353-65. 